\title{
Prise En Charge Des Dermo-Hypodermites Nécrosantes Au Centre Hospitalier Régional De Diffa, Niger
}

\author{
Ousseini Adakal, \\ Maman Bachir Abdoulaye,
}

Université Dan Dicko DanKoulodo de Maradi, Faculté des sciences de la santé, Département de chirurgie et spécialités chirurgicales, Centre

Hospitalier Régional de Maradi, Niger

\section{Harissou Adamou,}

Ibrahim Amadou Magagi,

Université de Zinder, Faculté des sciences de la santé, Département de chirurgie et spécialités chirurgicales, Hôpital National de Zinder, Niger

\section{Mohamed Rouga,}

Département de chirurgie et spécialités chirurgicales,

Centre Hospitalier Régional de Maradi, Niger

Mahamadou Doutchi,

Université de Zinder, Faculté des sciences de la santé, Service

d'infectiologie, Hôpital National de Zinder, Niger

\section{Mahaman Laouali Harouna, Oumarou Amadou,}

Université Dan Dicko DanKoulodo de Maradi, Faculté des sciences de la santé, Service Infectiologie, Centre Hospitalier Régional de Maradi, Niger

\section{Biraima Ahamadou,}

Université Dan Dicko DanKoulodo de Maradi, Faculté des sciences de la santé, Département de Médecine et spécialités médicales, Centre Hospitalier Régional de Maradi

\section{Onuoha Christopher,}

Université Dan Dicko DanKoulodo de Maradi, Faculté des sciences de la santé, Département de chirurgie et spécialités chirurgicales,

Centre Hospitalier Régional de Diffa

\section{Lassey James Didier,}

Sani Rachid,

Université Abdou Moumouni de Niamey, Faculté des sciences de la santé, Département de chirurgie et spécialités chirurgicales, Hôpital National de Zinder, Niger 


\section{Résumé}

Introduction : la dermo-hypodermite nécrosante (DHN) est une affection redoutable, responsable d'une morbi-mortalité importante constituant un véritable problème de santé publique dans la région de Diffa. L'objectif de cette étude est décrire la prise en charge des DHN dans le service de chirurgie du Centre Hospitalier Régional (CHR) de Diffa. Patients et méthodes : Il s'est agi d'une étude rétrospective descriptive, menée dans le service de chirurgie du CHR de Diffa, et incluant les dossiers des cas de DHN, pris en charge de Janvier 2017 à Décembre 2018. Résultats : 132 cas ont été enregistrés. L'âge médian était de 25 ans (extrêmes : 10 jours et 87 ans). Tous les patients ont bénéficié d'une antibiothérapie et dans $91,8 \%$ un traitement chirurgical était nécessaire. Le nombre de passage au bloc opératoire était compris entre 2 et 36 . Une greffe de peau a été réalisée dans $25,7 \%$ des cas. Le taux d'amputation de membre était de $9,1 \%$. La durée moyenne de séjour hospitalier était de 32,3 jours (extrêmes : 17 jours et 189 jours). La mortalité intra hospitalière était de 3,8\% $(\mathrm{n}=5)$. Conclusion: la dermo-hypodermite nécrosante est une affection grave, fréquente dans la région de Diffa dont la prise en charge médico-chirurgicale est lourde, grevée d'un mauvais pronostic. Un accent devrait être mis sur la recherche étiologique et le contrôle des facteurs prédisposant et aggravant de la maladie.

Mots clés : Dermo-Hypodermite, Nécrose, Diffa, Niger 


\title{
Management of Necrotizing Soft Tissue Infections in Regional Hospital of Diffa, Niger
}

\author{
Ousseini Adakal, \\ Maman Bachir Abdoulaye,
}

Université Dan Dicko DanKoulodo de Maradi, Faculté des sciences de la santé, Département de chirurgie et spécialités chirurgicales, Centre

Hospitalier Régional de Maradi, Niger

\section{Harissou Adamou,} Ibrahim Amadou Magagi,

Université de Zinder, Faculté des sciences de la santé, Département de chirurgie et spécialités chirurgicales, Hôpital National de Zinder, Niger

\section{Mohamed Rouga,}

Département de chirurgie et spécialités chirurgicales,

Centre Hospitalier Régional de Maradi, Niger

\section{Mahamadou Doutchi,}

Université de Zinder, Faculté des sciences de la santé, Service d'infectiologie, Hôpital National de Zinder, Niger

\section{Mahaman Laouali Harouna, Oumarou Amadou,}

Université Dan Dicko DanKoulodo de Maradi, Faculté des sciences de la santé, Service Infectiologie, Centre Hospitalier Régional de Maradi, Niger Biraima Ahamadou,

Université Dan Dicko DanKoulodo de Maradi, Faculté des sciences de la santé, Département de Médecine et spécialités médicales, Centre Hospitalier Régional de Maradi

\section{Onuoha Christopher,}

Université Dan Dicko DanKoulodo de Maradi, Faculté des sciences de la santé, Département de chirurgie et spécialités chirurgicales,

Centre Hospitalier Régional de Diffa

Lassey James Didier,

Sani Rachid,

Université Abdou Moumouni de Niamey, Faculté des sciences de la santé, Département de chirurgie et spécialités chirurgicales, Hôpital National de Zinder, Niger 
Abstract

Introduction: Necrotizing dermo-hypodermatitis (NHD) is a dreaded condition, responsible for a significant morbi-mortality constituting a real public health problem in the region of Diffa. The objective of this study is to describe the management of NHD in the surgical department of the Regional Hospital Center (CHR) of Diffa. Patients and methods: This was a descriptive retrospective study, conducted in the surgery department of the CHR of Diffa, and including the records of NHD cases, taken in charge from January 2017 to December 2018. Results: 132 cases were recorded. The median age was 25 years (extremes: 10 days and 87 years). All patients received antibiotic therapy and in $91.8 \%$ of cases surgical treatment was required. The number of visits to the operating theatre ranged from 2 to 36. A skin graft was performed in $25.7 \%$ of cases. The limb amputation rate was 9.1\%. The average length of hospital stay was 32.3 days (extremes: 17 days and 189 days). Intra-hospital mortality was $3.8 \% \quad(n=5)$. Conclusion: Necrotizing dermo-hypodermatitis is a serious condition, frequent in the region of Diffa, with a poor prognosis and heavy medical and surgical management. Emphasis should be placed on etiological research and control of predisposing and aggravating factors of the disease.

Keywords: Dermo-Hypodermitis, Necrotizing, Diffa, Niger

\section{Introduction}

Affection redoutable, connue sous la nomenclature anglo-saxonne de «necrotizing soft tissue infections «(NSTI), les dermo-hypodermites nécrosantes (DHN) regroupent un ensemble divers de lésions intéressant les parties molles dont le dénominateur commun est la nécrose tissulaire par opposition aux formes non nécrosantes (Stevens L et al, 2005). Elles sont responsables d'une morbi-mortalité importante (Stevens L et al, 2005). Tout retard diagnostique aggrave le pronostic nécessitant une chirurgie plus agressive voire des besoins de reconstruction et parfois des amputations de membres. Le traitement est lourd avec souvent recours aux soins intensifs (Emmanuel L et al 2016, Bilton BD et al 1998). L'incidence de cette affection est faible dans les pays développés : 4,8/1million d'habitant/an en France ; 4/100 000 habitant en Norvège et 5/1000000 personnes/année aux États-Unis (Majeski J et al 1997, Sbdian E et al 2014). Au Niger, cette affection n'est pas répertoriée dans les registres statistiques ni dans la littérature scientifique. C'est pourquoi cette étude se propose d'évaluer la prise en charge des DHN dans le service de chirurgie du CHR Diffa en décrivant les aspects épidémiologiques, cliniques, thérapeutiques et pronostiques. 


\section{Patients et méthode}

Il s'est agi d'une étude rétrospective descriptive, menée dans le service de chirurgie du CHR Diffa, et couvrant la période du 1er Janvier 2017 au 31 Décembre 2018 (2 ans). Etaient inclus dans l'étude, les dossiers des cas de DHN quelque soit l'étiologie bactérienne, admis et pris en charge dans ledit service durant la période d'étude. Les cas de DHN étaient pris en charge gratuitement à travers un partenariat avec un organisme international œuvrant dans le cadre de l'urgence humanitaire dans la région de Diffa. Les critères de non-inclusion concernaient les cas admis ayant refusé leur consentement pour un débridement chirurgical sous anesthésie ou une amputation d'emblée. Les variables étudiées étaient : l'âge, le sexe, la provenance, la localisation des lésions, les facteurs de gravité, le traitement et le pronostic. Les facteurs de gravité étaient définis selon le Score d'Anaya.

Tableau I : Facteurs de gravité des dermo-hypodermites nécrosantes selon le Score d'Anaya (Anaya DA et al, 2009).

\begin{tabular}{|c|c|c|}
\hline Variables à l'admission & & Points \\
\hline $\begin{array}{c}\text { Fréquence cardiaque }>110 \\
\text { battements /minute }\end{array}$ & & 1 \\
\hline Température $<36^{\circ} \mathrm{C}$ & & 1 \\
\hline Créatininémie $>1,5 \mathrm{mg} / \mathrm{dL}$ & & 1 \\
\hline Leucocytes $>40.000 / \mathrm{mL}$ & & 3 \\
\hline Hématocrite $>50 \%$ & & 3 \\
\hline Age $>50$ ans & & 3 \\
\hline Classe & $\begin{array}{l}\text { Nombre des } \\
\text { points }\end{array}$ & Mortalité (\%) \\
\hline I & $0-2$ & 6 \\
\hline II & $3-5$ & 24 \\
\hline III & $\geq 6$ & 88 \\
\hline
\end{tabular}

La prise en charge était basée sur le bilan général à la fois clinique et biologique et l'évaluation de l'état local du patient à l'admission. Le traitement initial était à la fois local (soins locaux au sérum physiologique, débridement chirurgical) et général (antibiothérapie, antalgiques, transfusion sanguine). L'antibiothérapie était empirique intraveineuse pendant 5 à 10 jours. Les associations d'antibiotiques souvent utilisées étaient : céfazoline+métronidazole, ceftriaxone + métronidazole, avec ou sans la gentamycine en cas de sepsis sévère. Puis il était procédé à un relai per os pour une durée de 7 à 21 jours avec Cloxacilline + Métronidazole ou Amoxicilline+Acide Clavulanique + Métronidazole.

Le traitement chirurgical était conduit sous anesthésie générale ou locorégionale (rachianesthésie) et consistait en une nécrosectomie extensive des lésions avec fasciotomie étendue, hémostase soigneuse, nettoyage au sérum physiologique. Le pansement de type absorbant au sucre était réévalué dans les 48 heures et ce, jusqu'au bourgeonnement effectif des plaies. Toute 
évolution défavorable des plaies justifiait un nouveau parage ou selon le cas, une amputation. Après l'obtention d'un bourgeon de qualité selon l'étendue des plaies, une suture secondaire ou une greffe de peau était réalisée. La nutrition entérale ou parentérale n'était pas disponible pour les patients de même que l'immunothérapie et les caissons hyperbares.

\section{Résultats}

Durant la durée de l'étude, -132 cas de DHN ont été colligés sur un total de 1487 admissions soit une fréquence de 8,9\%. Le sexe masculin était majoritaire avec 59,1\% $(n=78)$ soit un sex-ratio de 1,41. La médiane d'âge était de 25 ans (extrêmes : 10 jours et 87 ans). Les patients des plus de 50 ans représentaient $41,7 \%(\mathrm{n}=55)$ et les tranches d'âge de 16 à 50 ans et de 0 à 15 étaient respectivement à 35,6\% (n=47) et 22,7\% (n=30). La majorité des patients provenaient des différents districts sanitaires de la région (Tableau II).

Tableau II: Répartition des patients selon la provenance

\begin{tabular}{lcc}
\hline Provenance & Effectif & Pourcentage (\%) \\
\hline Nigeria & 24 & 18,2 \\
Maine Soroa & 41 & 31,1 \\
N'guigmi & 37 & 28 \\
Diffa & 7 & 5,3 \\
Bosso & 12 & 9,1 \\
Goudoumaria & 8 & 6,1 \\
N'gourty & 3 & 2,3 \\
\hline Total & $\mathbf{1 3 2}$ & $\mathbf{1 0 0}$ \\
\hline
\end{tabular}

Les facteurs de risque de la maladie rapportés dans cette étude étaient la prise d'anti-inflammatoires non stéroïdiens (AINS) dans 112 cas (84,8\%), les échardes dans 19 cas $(14,4 \%)$, l'adénophlegmon dans 7 cas $(5,3 \%)$, le diabète dans 6 cas $(4,5 \%)$, le VIH dans 5 cas $(3,8 \%)$ et les traumatismes négligés sans fractures dans 4 cas $(3,03 \%)$. Les lésions concernaient essentiellement le tronc et les membres (Figure 1).

Les principales localisations retrouvées dans notre série sont représentées ciaprès :

Tableau III : Répartition des patients selon la localisation des lésions

\begin{tabular}{ccc}
\hline Localisations & Effectif & Pourcentage (\%) \\
\hline Cervico-céphaliques & 9 & 6,8 \\
Thoraco-abdominales & 12 & 9,1 \\
Périnéales & 23 & 17,4 \\
Membres thoraciques & 41 & 31,1 \\
Membres pelviens & 47 & 35,6 \\
\hline Total & $\mathbf{1 3 2}$ & $\mathbf{1 0 0}$ \\
\hline
\end{tabular}



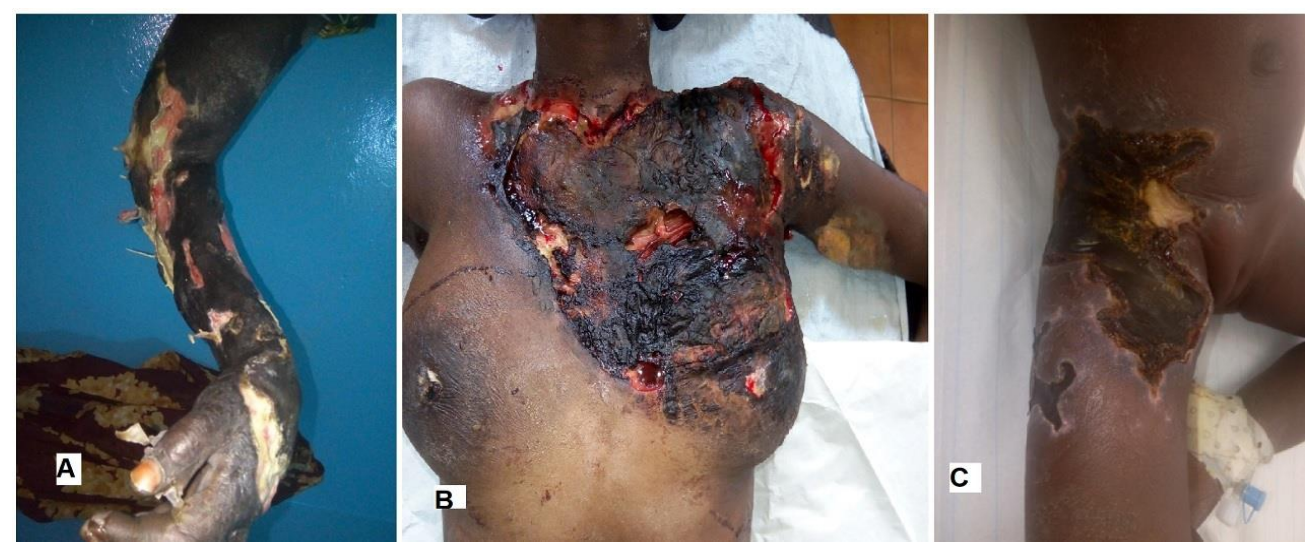

Figure 1-A : Membre thoracique gangréné, B : large plage de nécrose cutanée thoracique chez une accouchée récente, $\mathbf{C}$ : nécrose cutanée secondaire à un adénophlegmon inguinal droit.

Selon le score d'ANAYA; les classes I, II et III étaient respectivement à $36,5 \%, 54,5 \%$ et $9,1 \%$.

Les proportions des associations d'antibiotique utilisées étaient la céfazoline + métronidazole dans 78 cas $(59,1 \%)$, ceftriaxone +métronidazole dans 54 cas $(40,9 \%)$ et l'association avec la gentamycine dans 39 cas soit $29,5 \%$. Le relai per os de cloxacilline + métronidazole dans 105 cas soit $79,5 \%$ et l'amoxicilline acide clavulanique + métronidazole dans 27 cas soit $20,5 \%$. Le débridement chirurgical a été fait sous anesthésie générale avec pansement au sucre dans $78 \%(\mathrm{n}=103)$ des cas (Figure 2). L'anesthésie locorégionale était utilisée dans $22 \%$ des cas. La moyenne de réintervention était de 4,27 avec des extrêmes de 2 à 36 passages au bloc. La durée moyenne d'hospitalisation est de 32,3 jours avec des extrêmes de 17 et 189 jours. L'évolution des patients est rapportée dans le tableau IV.

Tableau IV : Évolution et Résultats des patients

\begin{tabular}{ccc}
\hline Résultats & Effectif & Pourcentage \\
\hline Greffe de peau & 34 & 25,8 \\
Cicatrisation dirigée & 28 & 21,2 \\
Sortie sur demande & 48 & 36,4 \\
Amputation & 17 & 12,9 \\
Décédé & 5 & 3,8 \\
\hline Total & $\mathbf{1 3 2}$ & $\mathbf{1 0 0}$ \\
\hline
\end{tabular}



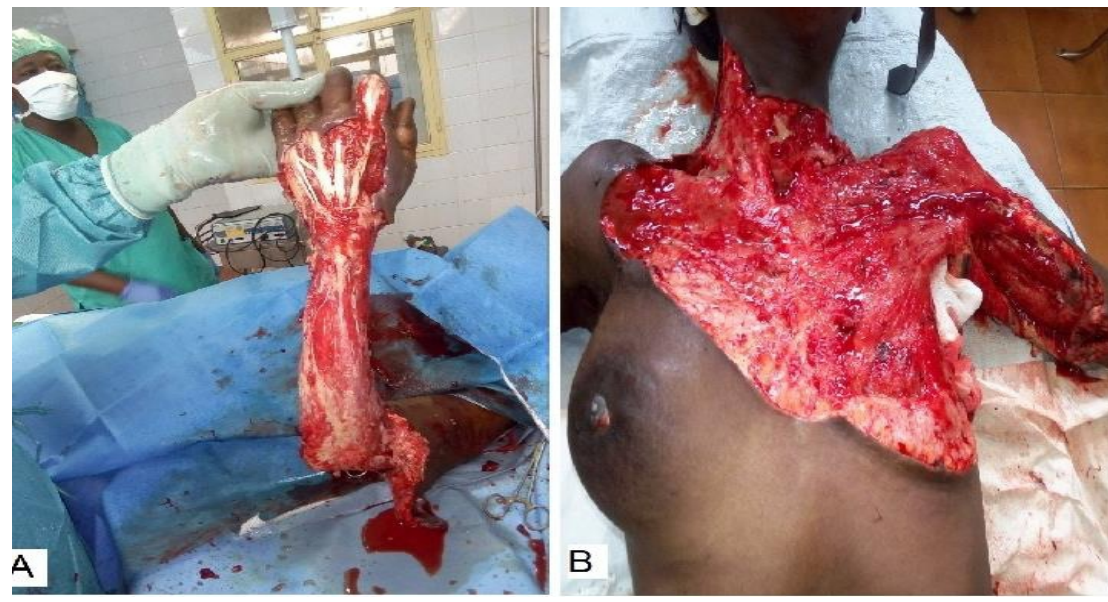

Figure 2- A : Aspect du membre supérieur après un débridement agressif, $\mathbf{B}$ : Atteinte thoracique chez une jeune accouché après débridement.

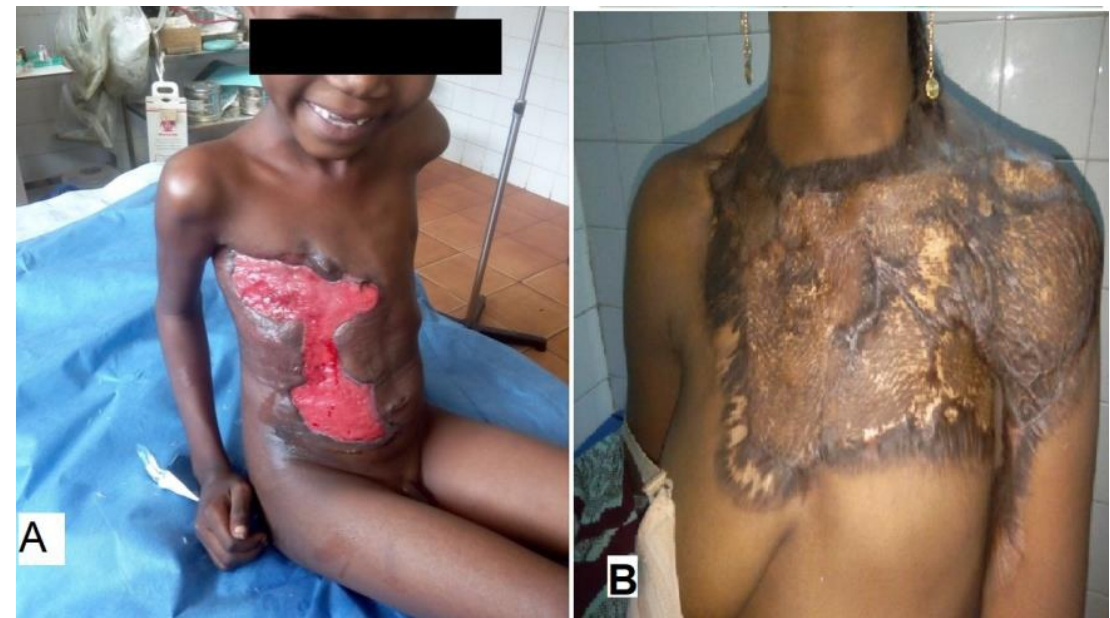

Figure 3-A: Atteinte thoraco-abdominale débridée en cours de cicatrisation dirigée, $\mathbf{B}$ : aspect de cicatrisation après greffe de peau.

\section{Discussion}

Les DHN résultent d'une infection progressive des parties molles due à des bactéries toxinogènes responsables d'une activation de cytokines inflammatoires avec survenue de phénomène de thromboses vasculaires entrainant une ischémie périphérique irréversible aboutissant à une nécrose tissulaire extensive (NM Garcia et al, 2018). Ce foyer local peut évoluer vers l'installation d'un sepsis ainsi que la survenue de défaillance multiviscérale en l'absence d'une prise en charge précoce. Contrairement aux pays développés (Sbdian E et al 2014, Arif N et al 2016, Anaya DA et al 2007), la prévalence relativement élevée des dermo-hypodermites nécrosantes dans notre contexte se justifierait par le faible niveau socio-économique combiné à l'insécurité en 
rapport avec l'insurrection Boko Haram qui prévaut depuis 2015. Cette insécurité étant responsable du déplacement des populations engendre du coup des problèmes d'hygiène ainsi qu'une dislocation des centres de soins les plus proches. En plus, ces facteurs expliqueraient le retard dans la consultation aggravant l'état général des patients avant l'admission. D'autre part, il est important de rappeler que le CHR de Diffa est le seul centre hospitalier de la région disposant d'une équipe chirurgicale assurant une prise en charge gratuite des cas de dermo-hypodermite. Ceci pourrait également expliquer cette prévalence intra-hospitalière élevée sans négliger les cas provenant du Nigeria frontalier.

Tous les âges sont concernés par cette affection avec une prédilection chez les plus jeunes témoignant d'un âge médian de 25 ans. Les facteurs de risques de cette affection dans notre contexte sont liés à l'automédication par les AINS et les piqures d'échardes négligées en milieu rural tandis que les auteurs occidentaux rapportaient les injections intraveineuses chez les toxicomanes, le diabète et l'éthylisme comme principaux facteurs de risque (Anaya DA et al 2012, Bosshardt TL et al 1996). En effet, la prise d'antiinflammatoires non stéroïdiens précédant l'épisode infectieux pouvait favoriser le passage d'un érysipèle ou d'une dermohypodermite bactérienne vers une DHN (C Souyri et al 2008).

La topographie des lésions dans notre série est polymorphe avec une prédominance des atteintes des membres qui semblent être les parties les plus exposées aux lésions par piqure d'échardes et autres traumatismes mineurs du fait du mode de vie rural des patients.

La prise en charge est médico-chirurgicale et doit être précédée d'une évaluation des facteurs de gravité dont l'intérêt pronostique a bien été démontré (Anaya DA et al 2009).

Pour ce qui est du traitement médical, l'antibiothérapie doit être empirique dirigée contre les aérobies et les anaérobies du fait qu'il ressort que 2/3 des dermo-hypodermites sont polymicrobiennes (Anaya DA et al 2007). Il est important de prendre en compte le niveau de résistance des germes aux céphalosporines de $3^{\text {ème }}$ génération rapporté dans une étude coréenne (Kim T et al 2019). Cette antibiothérapie permet d'aider à la réponse systémique contre l'infection, mais n'aurait aucun effet sur la nécrose tissulaire, véritable « gite à germes » (Anaya DA et al 2007). Le débridement chirurgical doit être fait en urgence et tout retard à la prise en charge chirurgicale augmenterait le risque de décès (Boyer A et AL 2009). Il s'agit d'une chirurgie agressive souvent débilitante et inesthétique, mais essentielle pour stopper la maladie et majorer la survie avec souvent nécessité d'un second-look dans les 48 heures qui suivent (NM Garcia et al 2018). L'oxygénothérapie hyperbare non disponible dans notre contexte aurait sans doute pu diminuer le nombre de passage au bloc opératoire et de pansement sous anesthésie générale 
(Wilkinson D et al, 2005). Aussi, d'autres auteurs ont insisté sur la place de la nutrition parentérale ou entérale ainsi que de la vitaminothérapie respectivement dans la réponse catabolique et l'aide à la guérison des plaies (Anaya D et al 2007, Phan HH et al 2010, Ustin JS et al 2011).

Le taux d'amputation de l'ordre de $12.9 \%$ s'expliquerait du fait du retard à la consultation et des formes graves, les indications d'amputation retenues dans la littérature étant l'atteinte d'un membre non viable ou présumé non fonctionnel après le débridement ou l'atteinte infectieuse extensive du membre mettant en jeu le pronostic vital (Anaya DA et al 2005). Aussi certains auteurs ont rapporté la réalisation de colostomies dans les atteintes périnéales péri-sphinctériennes (Kilic A et al 2001).

La guérison spontanée peut être obtenue aux dépens de l'allongement de la durée moyenne de séjour hospitalier. La principale cause d'abandon des soins est l'épuisement de la famille sur le plan social, matériel et psychologique. La greffe de peau, quoique refusée par certains patients, serait donc une alternative ainsi que toutes les autres thérapies (oxygénothérapie hyperbare, suppléments nutritionnels) permettant de diminuer la morbidité de cette affection.

La mortalité intrahospitalière faible de l'ordre de 3,8\% dans cette étude contrairement à celle rapportée dans la littérature (Barupal SR et al 2019) (6 à $76 \%$ ) serait en faveur de la rigueur de la prise en charge dans le service ainsi que la gratuité des soins

\section{Conclusion}

Relativement fréquentes, les DHN sont des affections dont la prise en charge est souvent lourde et pluridisciplinaire associant : chirurgien, infectiologue, anesthésiste-réanimateur, nutritionniste. Le traitement chirurgical est responsable de séquelles esthétiques et fonctionnelles souvent majeures. L'amputation de membre doit être considérée comme un traitement compte tenu des possibilités d'appareillage.

L'oxygénothérapie hyperbare ainsi que les compléments nutritionnels à fournir dans notre contexte peuvent permettre de réduire la morbi-mortalité des dermo-hypodermites nécrosantes.

Une étude avec une série plus importante, voire multicentrique, en mettant en évidence les souches bactériennes en cause viendrait à point nommé pour mieux comprendre cette affection.

\section{Conflit d'intérêts: aucun}

\section{References:}

1. Stevens DL, Bisno AL, Chambers EF, Everett ED, Dellinger P, Goldstein EJC, Gorbach SL, Hirschmann JV, Kaplan EL, Montoya JG, 
Wade JC (2005). Practice guidelines for the diagnosis and management of skin and soft-tissue infections. Clin Infect Dis 41:1373-1406.

2. Emmanuel L., Raphäl S., Yazine M. (2016). MAPAR P381-8.

3. Bilton BD, Zibari GB, McMillan RW, Aultman DF, Dunn G, McDonald JC (1998). Aggressive surgical management of necrotizing fasciitis serves to decrease mortality: a retrospective study. Am Surg ; 64: 397-400.

4. Majeski J, Majeski E. (1997). Necrotizing fasciitis: improved survival with early recognition by tissue biopsy and aggressive surgical treatment. South med J ; 90:1065-8.

5. SBIDIAN E. et al. (2014). Fasciites nécrosantes : première enquête épidémiologique française. Ann Dermatol Venereol, 141:297-98.

6. Anaya DA, Bulger EM, Kwon YS, et al. (2009). Predicting death in necrotizing soft tissue infections: a clinical score. Surg Infect (Larchmt) ; 10 (6):517-22.

7. NM. Garcia, J. Cai. (2018). Aggressive soft tissue infections. Surg Clin N Am ; 98 1097-1108.

8. Arif N, Yousf S, Vinnard C. (2016). Deaths from necrotizing fasciitis in the United States, 2003-2013. Epidemiology and infection; 144: 1338-44.

9. Anaya DA, Dellinger EP. (2007). Necrotizing soft-tissue infection: diagnosis and management Clin Infect Dis; 44(5):705-10.

10. Anaya DA, Dellinger EP. Necrotizing soft-tissue infection. In: Jong EC, Stevens DL, editors. (2012). Netter's infectious disease. 1st edition. Philadelphia: Saunders; 273-8.

11. Bosshardt TL, Henderson VJ, Organ CH Jr. (1996). Necrotizing softtissue infections. Arch Surg; 131(8):846-52.

12. C. Souyri et al. Severe necrotizing soft-tissue infections and NSAIDs. Clin Exp Dermatol, 33, 249-55

13. Kim T, Park SY, Kwak YG, Jung J, Kim MC, Choi SH, Yu SN, Hong HL, Kim YK, Park SY, Song EH, Park KH, Cho OH, Choi SH. (2019). Korean SSTI Study Group. Etiology, characteristics, and outcomes of community-onset necrotizing fasciitis in Korea: A multicenter study. PLoS One; 14(6):e0218668.

14. Boyer A. (2009). Influence of surgical treatment timing on mortality from necrotizing soft tissue infections requiring intensive care management Intensive Care Med ; 35:847-53

15. D. Wilkinson, D. Doolette. (2005). Hyperbaric oxygen treatmeent and survival from necrotizing soft tissue infection. Archivesof surgery; 139(12): 1339-45 
16. Phan HH, Cocanour CS. (2010). Necrotizing soft tissue infections in the intensive care unit. Crit Care Med; 38 (Suppl 9):S460-8.

17. Ustin JS, Malangoni MA. (2011). Necrotizing soft-tissue infections. Crit Care Med; 39(9):2156-62.

18. Anaya DA, McMahon K, Nathens AB, et al. (2005). Predictors of mortality and limb loss in necrotizing soft tissue infections. Arch Surg; 140:151-8.

19. Kilic A, Aksoy Y, Kilic L. (2001). Fournier's gangrene: etiology, treatment, and complications. Ann Plast Surg; 47:523-7.

20. Barupal SR, Soni ML, Barupal R. (2019). Factors Affecting Mortality Following Necrotizing Soft-Tissue Infections: Randomized Prospective Study. J Emerg Trauma Shock; 12(2):108-116. 